\title{
Subthalamic Nucleus Modulates Social and Anxogenic-like Behaviours
}

\section{AUTHORS}

\section{Jean-Michel Reymann \#}

- PhD, lecturer

- Université de Rennes 1, Laboratoire de Pharmacologie Expérimentale et Clinique, Faculté de Médecine, Rennes, France

- Centre d'Investigation Clinique, INSERM 0203 CIC-P, Unité de pharmacologie clinique, Université de Rennes 1, et hôpital universitaire de Pontchaillou, Rennes, France

\section{Florian Naudet \#}

- MD, Research Fellow

- Université de Rennes 1, Laboratoire de Pharmacologie Expérimentale et Clinique, Faculté de Médecine, Rennes, France

- Centre d'Investigation Clinique, INSERM 0203 CIC-P, Unité de pharmacologie clinique, Université de Rennes 1, et hôpital universitaire de Pontchaillou, Rennes, France

\# These authors contributed equally to this work.

\section{Morgane Pihan}

$-M D$

- Université de Rennes 1, Laboratoire de Pharmacologie Expérimentale et Clinique, Faculté de Médecine, Rennes, France

- Service des explorations fonctionnelles neurologiques, hôpital Pontchaillou, CHU de Rennes, rue Henri-Le-Guilloux, 35033 Rennes cedex 09, France

\section{Stephan Saïkali}

$-\mathrm{MD}, \mathrm{PhD}$ 
- Laboratoire d'Anatomopathologie, Faculté de Médecine de Rennes, CS34317, 2, avenue du Pr Léon Bernard, 35043 Rennes, France

\section{Bruno Laviolle}

- MD, PhD, lecturer

- Université de Rennes 1, Laboratoire de Pharmacologie Expérimentale et Clinique, Faculté de Médecine, Rennes, France

- Centre d'Investigation Clinique, INSERM 0203 CIC-P, Unité de pharmacologie clinique, Université de Rennes 1, et hôpital universitaire de Pontchaillou, Rennes, France

\section{Danièle Bentué-Ferrer}

- PhD, lecturer

- Université de Rennes 1, Laboratoire de Pharmacologie Expérimentale et Clinique, Faculté de Médecine, Rennes, France

\section{CORRESPONDING AUTHOR}

\section{Florian Naudet}

Service de Pharmacologie Clinique - CIC Inserm 0203

CHU de Rennes - Université de Rennes 1

Hôpital Pontchaillou - 2, rue Henri Le Guilloux, 35033 RENNES Cedex 9

Tél : +33 685836083, Fax : +33 299283722

E-mail: floriannaudet@gmail.com 


\section{ABSTRACT (228 words)}

In Parkinson's disease, global social maladjustment and anxiety are frequent after subthalamic nucleus (STN) stimulation and are generally considered to be linked with socio-familial alterations induced by the motor effects of stimulation. We hypothesized that the STN is per se involved in these changes and aimed to explore the role of STN in social and anxogenic-like behaviours using an animal model.

19 male Wistar rats with bilateral lesions of the STN were compared to 26 sham-lesioned rats by synchronizing an ethological approach based upon direct observation of social behaviours and a standardised approach, the elevated plus-maze (EPM). Comparisons between groups were performed by a Mann-Whitney-Wilcoxon test.

Lesioned rats showed impairments in their social $(p=0.05)$ and aggressive behaviours with a diminution of attacking $(p=0.04)$ and chasing $(p=0.06)$. In the EPM, concerning the open arms, the percentage of distance, time, inactive time, and entry were significantly decreased in lesioned rats $(p=0.02, p=0.01$, $p=0.04$ and $p=0.05$ ). The time spent in non-protected head dips was also diminished in the lesioned rats $(p=0.01)$.

These results strongly implicate the STN in social behaviour and anxogenic-like behaviour. In human, since DBS induces changes in the underlying dynamics of the stimulated brain networks, it could create an abnormal brain state in which anxiety and social behaviour are altered. These results highlight another level of complexity of the behavioural changes after stimulation.

Key Words: Basal ganglia, lesion, rats, social behaviour, anxiety 


\section{INTRODUCTION}

Slight to severe global social maladjustment is frequent in patients suffering from Parkinson Disease (PD) after subthalamic nucleus (STN) deep brain stimulation. In these patients, the motor improvement after surgery is followed in some cases by the deterioration of conjugal relationships which are triggered or aggravated postoperatively by the emergence of severe anxiety [1-2]. It has been hypothesised that these interpersonal conflicts were in line with an emergent property of systemic interactions between the patients, who suddenly regained their autonomy, and their devoted spouses, who lost their function after years of disease related compassion and care. It has also been hypothesised that such psychological side effects were due to sudden reduction in dopamine replacement therapy inducing a dopaminergic deficit in associativo-limbic areas of the brain [3].

Nevertheless, if the STN is a part of the basal ganglia which is well known to be involved in extrapyramidal control of movement [4-6], it is also linked with structures involved in the control of nonmotor behaviours via different neural associative and limbic circuits [7]. These functional networks may be of interest in behavioural regulation and the STN deep brain stimulation (DBS) could be per se involved in $1 /$ social maladjustment and $2 /$ emergence of anxiety. Moreover, psychological side effects described with DBS should differ regarding electrode target placement within different STN subterritories. Despite the number of STN subdivisions and their anatomical localisation is still uncertain [8], it has been suggested that the ventral part of the STN is closely involved in the emotional network [9]. While the neuronal bases of the effects of DBS remain unclear [10], it was hypothesised that $1 /$ changes in the underlying dynamics of the stimulated brain networks may represent the core mechanism of DBS and $2 /$ those basic dynamical changes can be achieved via activation, inhibition, or lesion [11]. Concerning STN lesions in humans, subthalamotomy could be a stereotactic surgery performed in patients suffering from PD in circumstances when deep brain stimulation is not viable [12]. Only one small prospective study including 10 patients found no change concerning anxiety following subthalamotomy [13] and, to our knowledge, no previous study has investigated its impact upon human social behaviour.

Thus, animal models could provide an opportunity for experimental manipulations that are not possible in human and can allow for exploring our hypotheses. To our knowledge, there was no previous investigation of consequences upon social behaviour of STN inactivation carried out in animals.

Thus, this study aimed to explore the role of STN on rat's social and anxogenic-like behaviours by synchronizing two complementary methods: 1) an ethological approach based upon direct observation of a wide range of social behaviours [14], and 2) a standardised approach using the elevated plus-maze (EPM), a "gold standard" test to explore anxiety like behaviours [15]. 


\section{MATERIAL AND METHODS}

\section{Animals}

Adults, male Wistar rats (CERJ), weighing 200-300 $\mathrm{g}$ at the beginning of the experiments, were kept 4 per cage in an approved animal house at $22 \pm 2{ }^{\circ} \mathrm{C}$, in 12-h light/dark cycle (light on at $0800 \mathrm{~h}$ ). Tap water and food pellets were available ad libitum. The animals were familiarized with the animal house at least ten days before surgery. The researchers are approved by the Veterinary department of the French Ministry of Health (authorization number 35-01). All animal experiments were carried out in accordance to the European Communities Council Directive (86/609/EEC). The care provided to them before, during and after the protocol was in compliance with ethical standards and good laboratory practices.

\section{Surgical procedures}

The animals were anesthetized with $350 \mathrm{mg} / \mathrm{kg}$ chloral hydrate injected intraperitoneally and secured into a Kopf stereotaxic apparatus (Phymep, Paris, France). Lesioned rats received bilateral injections of ibotenic acid $(9.4 \mu \mathrm{g} / \mu \mathrm{L}(53 \mathrm{mM})$, in $0.1 \mathrm{M}$ phosphate-buffered saline, $6.5<\mathrm{pH}<7)$. Sham-operated rats received vehicle alone ( $0.1 \mathrm{M}$ phosphate-buffered saline). Initially, the volume injected was $0,5 \mu \mathrm{L}$ per side and was slowly infused over 3 min using a 10- $\mu$ l Hamilton syringe. The needle was left in place for three min after completion of the injection. Nevertheless, as a small proportion of rats showed substantial brain lesions after histological verification, it was decided to inject $1 \mu \mathrm{L}$ per side.

The coordinates targeting the STN were determined according to Paxinos Watson's atlas [16] and were measured from bregma: anterior/posterior, - $3.7 \mathrm{~mm}$; lateral, $\pm 2.4 \mathrm{~mm}$; dorsal/ventral - $8.8 \mathrm{~mm}$ (from skull). Postoperatively, rats were housed individually in clear square plastic cages.

\section{Behavioural testing}

The same observer performed all the following behavioral testing.

\section{Open-field}

At day 12 after surgery, rats were individually placed in Plexiglas chambers $(45 \times 45 \times 30 \mathrm{~cm})$ at a light intensity of $30 \mathrm{~lx}$. Their spontaneous motor activity was measured min per min for a total duration of 10 
min, using a movement analysis system (Bioseb ${ }^{\circledR}$, Chaville, France), which counts movements by scanning $2 \times 16$ pairs of infrared photobeams on the side walls and allows for measuring spontaneous activity in the open field in lower position and in upper position. Furthermore, as all animals were placed in the open field, this phase allowed for habituation to the testing apparatus used in social interactions test.

\section{Social interaction test}

The general design of the social interaction tests was adapted from File et al. [17], Schneider et al. [1819], and Rudebeck et al. [20]. Social interactions were assessed at three different time points (day 13, 14 and 15 after surgery) in the open field (under the conditions described above) where rats were exposed to an unfamiliar social partner for $10 \mathrm{~min}$. Lines on the tail were drawn on sham and lesioned rats in order to recognize them from their congeners.

Partners used in social interaction tests were free of any surgical intervention. The test area was cleaned with a $5 \%$ alcohol solution after each trial. The two rats were recorded with a digital video camera and sequential elements of behavior were analyzed manually by a trained and blinded observer. The following behavioral elements were quantified (mean number of occurrences during the three sessions) in the experimental rats (sham or lesioned): non social behaviors, social behaviors and aggressive behaviors. Behaviors were also categorized as dominant or submissive. A detailed description of these behaviors is given in table 1 .

\section{Elevated plus-maze (EPM)}

This test was performed at day 16 after surgery. The EPM was made of wood and painted black. The apparatus consisted of two opposite open arms $(45 \times 10 \mathrm{~cm})$ without side walls and two enclosed arms $(45 \times 10 \times 30 \mathrm{~cm})$ with sides and end walls, extending from a central square $(10 \times 10 \mathrm{~cm})$. The maze was elevated to the height of $60 \mathrm{~cm}$ from the floor and placed in a moderately lit room (30 $\mathrm{lx}$ as measured at the centre of the maze). At the test onset, the animals were placed at the centre of the EPM, facing towards an open arm.

The Video-Track ${ }^{\circledR}$ system used for recording the animal behaviors was composed of a roof-handled camera connected to a computer. This apparatus measured activity or inactivity time and distances covered in each part of the maze in open or closed arms or center, minute per minute, for five minutes. The five following conventional parameters were recorded: total distance covered, number of entry in the open arms, percentage of distance and of time spent in the open arms, and percentage of inactive time spent in the open arms compared to total inactive time spent in the EPM as a whole. Furthermore, 
to minimize the possibility false-negative results [21], three ethological parameters were recorded on videotape and analyzed by a person not involved in the experimental procedure. The time devoted to the following behavioral activities was then recorded: 1) risk assessment (RA), the rat exiting an enclosed arm with the forepaws and head only and investigating the surroundings, not necessarily accompanied by body stretching; 2) protected head dips (PHD), the rat stretching to dip its head into the open space and observe the environment, the rear part of the body remaining in a closed arm; and 3) non-protected head dips (NPHD), the rat dipping its head into the open space and observing the environment, the body in an open arm.

The EPM was cleaned between the experiments with a $5 \%$ alcohol solution.

\section{Histological verification of lesion}

At the end of the experiments, the animals were anesthetized with $350 \mathrm{mg} / \mathrm{kg}$ chloral hydrate injected intraperitoneally and decapitated.

The brains were removed and placed in $3.7 \%$ formaldehyde for 48 hours, processed and embedded in paraffin. Coronal sections were cut at a thickness of $5 \mu \mathrm{m}$ every $50 \mu \mathrm{m}$ in the NST area and stained with haematoxylin-eosin-saffron (HES). After staining, slides were observed under an optical microscope (Nikon 80i). An experimenter unaware of the animal's behavioural performance performed an histological verification of the microinjection site. Lesions were described with reference to Paxinos and Watson's stereotaxic rat brain atlas [16]. Measures were performed using the software Image $₫$ (Mac Biophotonics). Only rats with bilateral lesions of the STN were kept in the analysis and the absence of lesions was verified in sham rats.

\section{Statistical analysis}

Statistical analysis was performed with SAS statistical software, version 9.2 (SAS Institute, Cary, NC, USA) and R (R Development Core Team). Results are presented as arithmetic mean and standard error of the mean ( $m \pm$ s.e.m.). The level of significance was set at 0.05 .

Comparisons between groups were performed by a Mann-Whitney-Wilcoxon test. To adjust our comparisons between social behaviour of lesioned and sham rats on the confounding effect of motility, a general linear model was used. The dependent variable was the number of behaviours; the two explanatory variables were the group (reference: lesioned) and the spontaneous activity in lower position in the open field (mobility) at day 12.

\section{RESULTS}


Animals

Among 48 operated rats, 19 had bilateral lesions of the STN and were kept in the analysis. Indeed, for the other 29 rats no lesion was observed for the STNs and lesions interested other parts of the brain. 26 sham rats were also included in the analysis. Two lesioned rats and 3 sham-operated rats did not participate to social interaction test due to technical problems (cd-roms were physically damaged).

Histology

Figure 1 shows a representative section from a sham control and an animal with an STN lesion. STN lesioned animals showed a loss of neurons that was mainly restricted to the STN, with little damage to structures outside the STN.

\section{Behavioural testing}

\section{Open-field}

No difference was observed between lesioned and sham-operated rats concerning their spontaneous activity in the open field in lower position $4490 \pm 459$ counts versus $4736 \pm 239$ counts respectively $(p=0.25)$ or in upper position $870 \pm 135$ versus $1006 \pm 90$ counts respectively $(p=0.19)$.

\section{Social interaction test}

While their non social behaviour was not significantly impaired (108.1 \pm 7.7 vs $97.4 \pm 4.8, p=0.38$ ), lesioned rats showed impairments in their social (71.2 \pm 7.9 vs $103.0 \pm 11.2, p=0.05)$ and aggressive behaviours (74.7 \pm 6.0 vs $90.9 \pm 6.2$, trend toward statistical significance: $p=0.06$ ) when compared to sham-operated rats. Table 2 presents the detail of impaired behaviours.

When adjusting on the confounding effect of motility, these differences concerning social $(p=0.03)$ and aggressive behaviours (with a trend to statistical significance, $p=0.08$ ) were preserved. Moreover, the multivariate analysis concerning non social behaviour suggested that lesioned rats presented more nonsocial behaviours $(p=0.04)$. Results of these models are presented in figure 2. Any outliers were identified and removed: while the results were unchanged for social and aggressive behaviours the difference between groups concerning non-social behaviours became non-significant making this result less robust than the two others. 


\section{Elevated-plus maze}

Results of conventional and ethological parameters are shown in figure 3. Concerning the conventional parameters, whereas the total distance covered in the whole EPM was not statistically significant different between lesioned and sham-operated groups, the percentage of distance covered in the open arms, the percentage of time in the open arms, and the percentage of inactive time spent in the open arms, were significantly decreased in the lesioned rats $(p=0.02, p=0.01$ and $p=0.04$, respectively). Although not significant, the number of entry in the open arms also tended to decrease $(p=0.052)$.

Concerning the three ethological parameters, only the time spent in non-protected head dips was significantly diminished in the lesioned rats $(p=0.01)$.

\section{DISCUSSION}

\section{Summary of evidence}

This study corroborated our hypotheses by showing that the STN is implicated in social behaviour regulation and in anxogenic-like behaviors. Lesioned rats presented impairments in social and aggressive behaviours whereas their non social behaviours were not affected. These findings did not result from changes in motor activity since the decrease in social interests persisted after adjustment on rats' motility. Lesioned rats were less aggressive in the whole with no difference between dominant and submissive behaviors.

Lesioned rats also showed more anxogenic-like behaviours in the EPM, as illustrated by an increase of the time spent in the enclosed arms [15]. This result is in line with previous findings in human after deep brain stimulation where an increase in anxiety was observed postoperatively [1, 22-24]. However it contrasts with the therapeutic effect of STN stimulation in obsessive compulsive disorder [25] which is a well known anxious disorder and with the only and weak evidence upon 10 patients who underwent subthalamotomy who did not experience change concerning anxiety [13]. Concerning animals' models, our result contrasts with Klein et al.'s and Creed et al.'s previous findings in which a lesion of the STN or a STN DBS did not affect anxogenic-like behaviours [26-27]. An handling for about 2 min daily for 7 days prior to the beginning of the behavioural experiment in Klein's study as well as an extensive daily handling (> 12 weeks) in Creed's study could account for the observed differences. Indeed, one of our previous experiment suggested that handling has precisely an anxiolytic-like effect [28]. Moreover, in Klein's study, behavioural experiments started 6 weeks after surgery whereas in our study the EPM was used much earlier (16 days after surgery). All together, these results suggest a possible time-effect of STN inactivation upon anxious behaviours with possible adaptative mechanisms of plasticity controlling anxiety and raise new perspectives concerning a longitudinal analysis of lesioned rats' behaviours. 
Further experiments should consider testing after more extensive handling and/or testing at different time points.

\section{Perspective}

Pharmacologists usually consider social interaction test as a test of anxiety [17], based on a natural form of behaviour by pairs of male rats in different kind of social interactions. An increase in social interactions is considered to reflect an anxiolytic-like effect and a decrease indicates an anxiogenic-like effect. It has been shown to be sensitive to changes in anxiety generated by nonpharmacological factors [17]. Nevertheless, results observed in social interaction tests have to be put into perspective. Indeed, these tests explore experimental rat's behaviour in the presence of a congener but also the congener's behaviour in the presence of the experimental rat, and the occurrence of a particular non social act may itself be a social phenomenon [14]. Indeed, social behaviour of the rats is not only the reflect of their performances in other models of anxiety [29-30] and social interaction tests do not solely explore emotional processes but a more global level of functioning including motors, cognitive and motivational processes.

From a functional point of view the STN, with its three different sub territory (limbic, associative and motor areas), has precisely an integrative function at the interface between motivation, cognition and action. It is a small lens-shaped structure which modulates the neurotransmission in limbic regions of the brain [31] involved in anxiety regulation. Experimentally, non-motor role of the STN has been confirmed in rats, particularly for impulsive behaviours [32-33], motivational functions [34] and attentional performances [35]. STN also modulates differently the effects of natural rewards, like food, or drug abuse, like cocaine, on behaviour [36]. STN HFS also transiently impairs attention in a visual attention task [37]. It was shown that inactivation of the rat STN evoked depressive like-behaviour in the forced swim test (Temel, et al 2007) and also in the learn helplessness test [26-27]. In human also, STN DBS adverse effects on cognition, behaviour, mood and emotions are well known [38-41].

All together, these modifications can alter social behaviour. Our study does not allow for discriminating the effect of each of these dimensions upon social behaviour but clearly describes in an ethological perspective, qualitative impairments of social behaviour as a potential result of these multiple alterations. Moreover, in humans, in PD, STN stimulation could affect Theory of Mind Network [42] and could be implicated in action observation and action understanding [43]. These modifications of social cognition might explain some of the behavioural disturbances related to STN stimulation in patients. Of course such stimulating hypotheses can not be studied in rodents [44] but it is noticeable that, in our model, only social behaviours where impaired whereas non social behaviours were preserved.

Limitations 
The extrapolation from a rat model to the clinical condition should be made with great caution. Firstly, our rat model with bilateral subthalamic lesions induced with ibotenic acid injections is not really representative of the clinical situation given the integrity of dopaminergic projections. Secondly, hudge behavioural differences in anxiety, exploration and social behaviour exist between species and even between different strains of a same species as it was shown for rats [29, 45]. Thus the robustness of the present findings should be tested in others animal models among which specific studies of social behaviour in monkeys should be of particular interest. It would allow for exploration of the different sub territory (limbic, associative and motor areas) of the STN [46]. Indeed the rat's STN is a very small structure in which specific investigation of its sub-territories is actually impossible.

\section{Conclusion}

The results of the present study strongly implicate the STN in social behaviour and anxogenic-like behaviour in rats. In human, since DBS induces changes in the underlying dynamics of the stimulated brain networks it could create an abnormal brain state in witch anxiety and social behaviour are affected. It does not mean that social maladjustment is not linked with adaptation difficulties and dramatic socio-familial modification induced by the motor effects of DBS, but it highlights another possible level of complexity of these behavioural changes after stimulation.

Other animal and human studies are needed to confirm robustness of these findings and to add a longitudinal perspective. If the implication of STN upon social behaviour is confirmed, a new challenge should be to develop interventions with less impact on these behavioural aspects or interventions which could help stimulated patients to cope with the dramatic changes in their social functioning.

\section{AKNOWLEDGEMENTS}

We would like to thank the Rennes 1 University UFR of Medicine for its financial support, Mrs Julie Querzerho and Mr Jean-Christophe Coudeyre for their valuable technical assistance and Mrs Claudine Naudet for revising the English. We thank Elsevier for granting us permission to reproduce a figure from "The Rat Brainin Stereotaxic Coordinates" COMPACT THIRD EDITION by George Paxinos and Charles Watson, Academic Press, Inc., 2007, ISBN: 0-12-547623-X.

The sponsor had no role concerning: design and conduct of the study; collection, management, analysis, and interpretation of the data; and preparation, review, or approval of the manuscript. 
Funding: Rennes 1 University UFR of Medicine; the sponsor had no role concerning: design and conduct of the study; collection, management, analysis, and interpretation of the data; and preparation, review, or approval of the manuscript.

\section{Conflict of interests:}

There are no conflicts of interest regarding this paper. All authors have completed the Unified Competing Interest form at http://www.icmje.org/coi_disclosure.pdf (available on request from the corresponding author) and declare that (1) No authors have support from any company for the submitted work; (2) R.J.M. P.M. L.B. B.F.D. have no relationships with any company that might have an interest in the submitted work in the previous 3 years; N.F. has relationships (board membership or Travel/accommodations expenses covered/reimbursed) with Servier, BMS, Lundbeck and Janssen who might have an interest in the work submitted in the previous 3 years ; (3) R.J.M. N.F. P.M. L.B. B.F.D. spouses, partners, or children have no financial relationships that may be relevant to the submitted work; and (4) none of the authors has any non-financial interests that may be relevant to the submitted work.

\section{Authors contributions:}

Jean Michel Reymann had full access to all of the data in the study and takes responsibility for the integrity of the data and the accuracy of the data analysis.

Conceived and designed the experiments: R.J.M. and B.F.D.

Performed the experiments: R.J.M. P.M. and B.F.D.

Analyzed the data: L.B. N.F.

Contributed reagents/materials/analysis tools: N.F.

Wrote the paper: N.F. R.J.M. and B.F.D.

Revised the paper critically for important intellectual content: P.M. L.B.

Final approval of the version to be published: R.J.M. N.F. P.M. L.B. B.F.D.

\section{ABBREVIATIONS}

PD: Parkinson Disease

STN: SubThalamic Nucleus

EPM: Elevated Plus Maze 


\section{REFERENCES}

[1] Houeto JL, Mesnage V, Mallet L, Pillon B, Gargiulo M, du Moncel ST, et al. Behavioural disorders, Parkinson's disease and subthalamic stimulation. J Neurol Neurosurg Psychiatry. 2002;72:701-7.

[2] Perozzo P, Rizzone M, Bergamasco B, Castelli L, Lanotte M, Tavella A, et al. Deep brain stimulation of subthalamic nucleus: behavioural modifications and familiar relations. Neurol Sci. 2001;22:81-2.

[3] Czernecki V, Schupbach M, Yaici S, Levy R, Bardinet E, Yelnik J, et al. Apathy following subthalamic stimulation in Parkinson disease: a dopamine responsive symptom. Mov Disord. 2008;23:964-9.

[4] Parent A, Hazrati LN. Functional anatomy of the basal ganglia. I. The cortico-basal ganglia-thalamocortical loop. Brain Res Brain Res Rev. 1995;20:91-127.

[5] Parent A, Hazrati LN. Functional anatomy of the basal ganglia. II. The place of subthalamic nucleus and external pallidum in basal ganglia circuitry. Brain Res Brain Res Rev. 1995;20:128-54.

[6] Utter AA, Basso MA. The basal ganglia: an overview of circuits and function. Neurosci Biobehav Rev. 2008;32:333-42.

[7] Temel Y, Blokland A, Steinbusch HW, Visser-Vandewalle V. The functional role of the subthalamic nucleus in cognitive and limbic circuits. Prog Neurobiol. 2005;76:393-413.

[8] Keuken MC, Uylings HB, Geyer S, Schafer A, Turner R, Forstmann BU. Are there three subdivisions in the primate subthalamic nucleus? Front Neuroanat. 2012;6:14.

[9] Peron J, Fruhholz S, Verin M, Grandjean D. Subthalamic nucleus: a key structure for emotional component synchronization in humans. Neurosci Biobehav Rev. 2013;37:358-73.

[10] McConnell GC, So RQ, Hilliard JD, Lopomo P, Grill WM. Effective deep brain stimulation suppresses low-frequency network oscillations in the basal ganglia by regularizing neural firing patterns. J Neurosci. 2012;32:15657-68.

[11] Mclntyre CC, Hahn PJ. Network perspectives on the mechanisms of deep brain stimulation. Neurobiol Dis. 2010;38:329-37.

[12] Alvarez L, Macias R, Lopez G, Alvarez E, Pavon N, Rodriguez-Oroz MC, et al. Bilateral subthalamotomy in Parkinson's disease: initial and long-term response. Brain. 2005;128:570-83.

[13] Bickel S, Alvarez L, Macias R, Pavon N, Leon M, Fernandez C, et al. Cognitive and neuropsychiatric effects of subthalamotomy for Parkinson's disease. Parkinsonism Relat Disord. 2010;16:535-9.

[14] Mackintosh J, Chance M, Silvermann A. The contribution of ethological techniques to the study of drug effects In: Iversen S, Snyder S, editors. Handbook of psychopgharmacology. New York: Plenum press; 1977. 
[15] Pellow S, Chopin P, File SE, Briley M. Validation of open:closed arm entries in an elevated plusmaze as a measure of anxiety in the rat. J Neurosci Methods. 1985;14:149-67.

[16] Paxinos G, Watson C. The Rat Brain in Stereotaxic Coordinates. New York: Academic Press; 1998.

[17] File SE, Seth P. A review of 25 years of the social interaction test. Eur J Pharmacol. 2003;463:3553.

[18] Schneider M, Schomig E, Leweke FM. Acute and chronic cannabinoid treatment differentially affects recognition memory and social behavior in pubertal and adult rats. Addict Biol. 2008;13:345-57.

[19] Schneider M, Koch M. Deficient social and play behavior in juvenile and adult rats after neonatal cortical lesion: effects of chronic pubertal cannabinoid treatment. Neuropsychopharmacology. 2005;30:944-57.

[20] Rudebeck PH, Walton ME, Millette BH, Shirley E, Rushworth MF, Bannerman DM. Distinct contributions of frontal areas to emotion and social behaviour in the rat. Eur J Neurosci. 2007;26:231526.

[21] Carobrez AP, Bertoglio LJ. Ethological and temporal analyses of anxiety-like behavior: the elevated plus-maze model 20 years on. Neurosci Biobehav Rev. 2005;29:1193-205.

[22] Dujardin K, Defebvre L, Krystkowiak P, Blond S, Destee A. Influence of chronic bilateral stimulation of the subthalamic nucleus on cognitive function in Parkinson's disease. J Neurol. 2001;248:603-11.

[23] Porat O, Cohen OS, Schwartz R, Hassin-Baer S. Association of preoperative symptom profile with psychiatric symptoms following subthalamic nucleus stimulation in patients with Parkinson's disease. $J$ Neuropsychiatry Clin Neurosci. 2009;21:398-405.

[24] Strutt AM, Simpson R, Jankovic J, York MK. Changes in cognitive-emotional and physiological symptoms of depression following STN-DBS for the treatment of Parkinson's disease. Eur $\mathrm{J}$ Neurol. 2012;19:121-7.

[25] Mallet L, Polosan M, Jaafari N, Baup N, Welter ML, Fontaine D, et al. Subthalamic nucleus stimulation in severe obsessive-compulsive disorder. N Engl J Med. 2008;359:2121-34.

[26] Creed MC, Hamani C, Nobrega JN. Effects of repeated deep brain stimulation on depressive- and anxiety-like behavior in rats: Comparing entopeduncular and subthalamic nuclei. Brain Stimul. 2012.

[27] Klein J, Winter C, Coquery N, Heinz A, Morgenstern R, Kupsch A, et al. Lesion of the medial prefrontal cortex and the subthalamic nucleus selectively affect depression-like behavior in rats. Behav Brain Res. 2010;213:73-81.

[28] Robert G, Drapier D, Bentue-Ferrer D, Renault A, Reymann JM. Acute and chronic anxiogenic-like response to fluoxetine in rats in the elevated plus-maze: modulation by stressful handling. Behav Brain Res. 2011;220:344-8. 
[29] Ramos A, Berton O, Mormede P, Chaouloff F. A multiple-test study of anxiety-related behaviours in six inbred rat strains. Behav Brain Res. 1997;85:57-69.

[30] Tonissaar M, Philips MA, Eller M, Harro J. Sociability trait and serotonin metabolism in the rat social interaction test. Neurosci Lett. 2004;367:309-12.

[31] Winter C, Lemke C, Sohr R, Meissner W, Harnack D, Juckel G, et al. High frequency stimulation of the subthalamic nucleus modulates neurotransmission in limbic brain regions of the rat. Exp Brain Res. 2008;185:497-507.

[32] Uslaner JM, Robinson TE. Subthalamic nucleus lesions increase impulsive action and decrease impulsive choice - mediation by enhanced incentive motivation? Eur J Neurosci. 2006;24:2345-54.

[33] Winstanley CA, Baunez C, Theobald DE, Robbins TW. Lesions to the subthalamic nucleus decrease impulsive choice but impair autoshaping in rats: the importance of the basal ganglia in Pavlovian conditioning and impulse control. Eur J Neurosci. 2005;21:3107-16.

[34] Baunez C, Humby T, Eagle DM, Ryan LJ, Dunnett SB, Robbins TW. Effects of STN lesions on simple vs choice reaction time tasks in the rat: preserved motor readiness, but impaired response selection. Eur J Neurosci. 2001;13:1609-16.

[35] Baunez C, Robbins TW. Bilateral lesions of the subthalamic nucleus induce multiple deficits in an attentional task in rats. Eur J Neurosci. 1997;9:2086-99.

[36] Baunez C, Dias C, Cador M, Amalric M. The subthalamic nucleus exerts opposite control on cocaine and 'natural' rewards. Nat Neurosci. 2005;8:484-9.

[37] Baunez C, Christakou A, Chudasama Y, Forni C, Robbins TW. Bilateral high-frequency stimulation of the subthalamic nucleus on attentional performance: transient deleterious effects and enhanced motivation in both intact and parkinsonian rats. Eur J Neurosci. 2007;25:1187-94.

[38] Biseul I, Sauleau P, Haegelen C, Trebon P, Drapier D, Raoul S, et al. Fear recognition is impaired by subthalamic nucleus stimulation in Parkinson's disease. Neuropsychologia. 2005;43:1054-9.

[39] Temel Y, Kessels A, Tan S, Topdag A, Boon P, Visser-Vandewalle V. Behavioural changes after bilateral subthalamic stimulation in advanced Parkinson disease: a systematic review. Parkinsonism Relat Disord. 2006;12:265-72.

[40] Vicente S, Biseul I, Peron J, Philippot P, Drapier S, Drapier D, et al. Subthalamic nucleus stimulation affects subjective emotional experience in Parkinson's disease patients. Neuropsychologia. 2009;47:1928-37.

[41] Peron J, Biseul I, Leray E, Vicente S, Le Jeune F, Drapier S, et al. Subthalamic nucleus stimulation affects fear and sadness recognition in Parkinson's disease. Neuropsychology. 2010;24:1-8. 
[42] Peron J, Le Jeune F, Haegelen C, Dondaine T, Drapier D, Sauleau P, et al. Subthalamic nucleus stimulation affects theory of mind network: a PET study in Parkinson's disease. PLoS One. 2010;5:e9919.

[43] Marceglia S, Fiorio M, Foffani G, Mrakic-Sposta S, Tiriticco M, Locatelli M, et al. Modulation of beta oscillations in the subthalamic area during action observation in Parkinson's disease. Neuroscience. 2009;161:1027-36.

[44] Baunez C, Yelnik J, Mallet L. Six questions on the subthalamic nucleus: lessons from animal models and from stimulated patients. Neuroscience. 2011;198:193-204.

[45] Rex A, Sondern U, Voigt JP, Franck S, Fink H. Strain differences in fear-motivated behavior of rats. Pharmacol Biochem Behav. 1996;54:107-11.

[46] Gubellini P, Salin P, Kerkerian-Le Goff L, Baunez C. Deep brain stimulation in neurological diseases and experimental models: from molecule to complex behavior. Prog Neurobiol. 2009;89:79123. 


\begin{tabular}{|c|c|}
\hline Behaviours & Detailed description \\
\hline \multicolumn{2}{|l|}{ Non social behaviours } \\
\hline Non social exploration & - Exploring the open field. \\
\hline Rearing & - Standing on its hind legs and appearing to be looking at something. \\
\hline Resting & - Passive behavior in which the rat does not move and has a relaxed body posture. \\
\hline Self-grooming & $\begin{array}{l}\text { - Licking or biting its own fur. } \\
\text { - Rubbing the forepaws over the head. }\end{array}$ \\
\hline \multicolumn{2}{|l|}{ Social behaviours } \\
\hline Contact behavior & $\begin{array}{l}\text { - Grooming (chewing and licking the partner's fur). } \\
\text { - Crawling over the social partner. }(+) \\
\text { - Crawling under the social partner. }(-)\end{array}$ \\
\hline Social exploration & $\begin{array}{l}\text { - Anogenital investigation (sniffing or licking the anogenital area of the social partner). } \\
\text { - Non-anogenital investigation (sniffing at any part of the partner's body, except the } \\
\text { anogenital area). }\end{array}$ \\
\hline Tail manipulation & - Grabbing and pulling the partner's tail. \\
\hline \multicolumn{2}{|l|}{ Aggressive behaviours } \\
\hline Pinning & $\begin{array}{l}\text { - The social partner is lying on its back with the experimental animal standing over him. }\left(^{+}\right) \\
\text {- The experimental animal is lying on its back with the social partner standing over him. }(-)\end{array}$ \\
\hline Attacking & $\begin{array}{l}\text { - Boxing. }(+) \\
\text { - Biting. }(+)\end{array}$ \\
\hline Chasing & - Rapide pursuits. (+) \\
\hline Evading & - Running, leaping or swerving away from the social partner. (-) \\
\hline
\end{tabular}

Table 1: detailed description of behavioral rating

(+) Dominant behavior

(-) Submissive behavior 


\begin{tabular}{|lccc|}
\hline & Lesioned $(\mathbf{n}=\mathbf{1 7})$ & Sham $(\mathbf{n}=\mathbf{2 3})$ & $\mathbf{p}$-value \\
Non social behaviours & $108.1 \pm 7.7$ & $97.4 \pm 4.8$ & 0.38 \\
Non social exploration & $54.6 \pm 1.2$ & $49.3 \pm 2.0$ & 0.15 \\
Rearing & $45.4 \pm 6.7$ & $36.9 \pm 3.0$ & 0.44 \\
Immobility & $15.7 \pm 3.0$ & $9.5 \pm 2.0$ & 0.18 \\
Self-grooming & $4.7 \pm 0.5$ & $6.3 \pm 1.1$ & 0.39 \\
Social behaviours & $71.2 \pm 7.9$ & $103.0 \pm 11.2$ & $\mathbf{0 . 0 5}$ \\
Contact behavior & $57.8 \pm 3.9$ & $72.4 \pm 5.4$ & $\mathbf{0 . 0 4}$ \\
Social exploration & $12.4 \pm 3.7$ & $26.5 \pm 5.3$ & 0.06 \\
Tail manipulation & $1.0 \pm 0.8$ & $4.1 \pm 1.3$ & $\mathbf{0 . 0 2}$ \\
Aggressive behaviours & $74.7 \pm 6.0$ & $90.9 \pm 6.2$ & 0.06 \\
Pinning & $9.1 \pm 1.3$ & $8.7 \pm 1.4$ & 0.45 \\
Attacking & $22.8 \pm 2.5$ & $29.5 \pm 2.2$ & $\mathbf{0 . 0 4}$ \\
Chasing & $39.1 \pm 3.1$ & $48.9 \pm 3.5$ & $\underline{0.06}$ \\
Evading & $3.7 \pm 0.6$ & $3.7 \pm 0.7$ & 0.62 \\
Submissive behaviours & $29.2 \pm 6.0$ & $45.6 \pm 7.7$ & 0.24 \\
Submissive non aggressive & $16.8 \pm 2.3$ & $16.7 \pm 2.2$ & 0.88 \\
Submissive aggressive & $12.4 \pm 4.5$ & $28.9 \pm 5.9$ & $\mathbf{0 . 0 2}$ \\
Dominant behaviours & $18.7 \pm 1.4$ & $25.9 \pm 2.8$ & 0.14 \\
Dominant non aggressive & $15.2 \pm 1.1$ & $17.5 \pm 1.7$ & 0.54 \\
Dominant aggressive & $3.5 \pm 1.0$ & $8.4 \pm 1.7$ & $\mathbf{0 . 0 5}$ \\
\hline
\end{tabular}

Table 2: Differences in social interaction test between lesioned and sham male rats. Results presented are the mean number of behaviours observed during test sessions and are presented in mean \pm s.e.m. $p$-value presented are $p$-values of univariate analyses. 

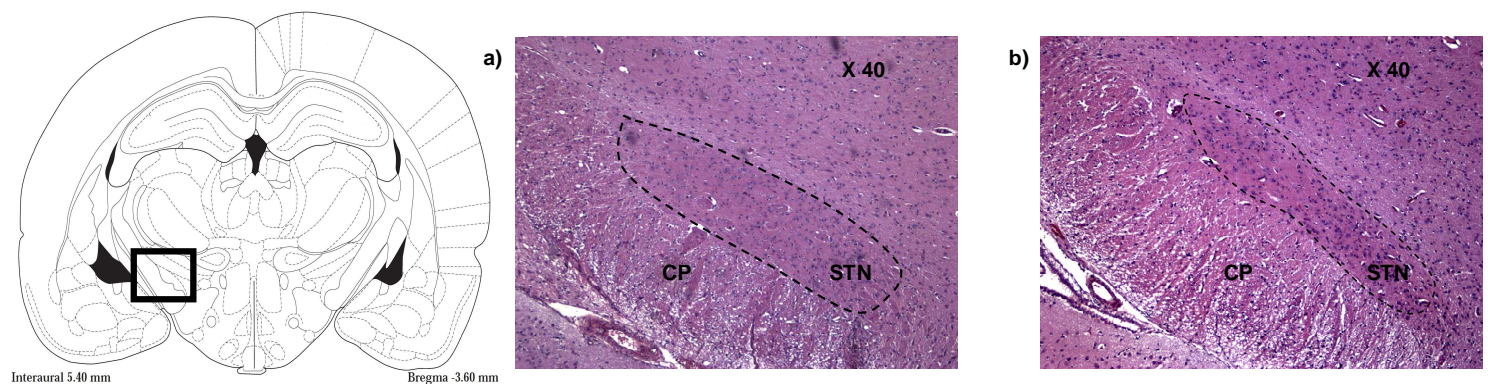

Figure 1: Sections of the left STN. Region of section is indicated on a schematic coronal image from Paxinos and Watson (reproduced with permission). Photomicrographs (X 40) of 2 representative haematoxylin-eosin-saffron stained sections showing the subthalamic nucleus (STN) bordering the cerebral peduncle $(\mathrm{CP})$ :
a) in a sham operated rat ;
b) in a lesioned rat (atrophy of the STN and significant cell loss). 

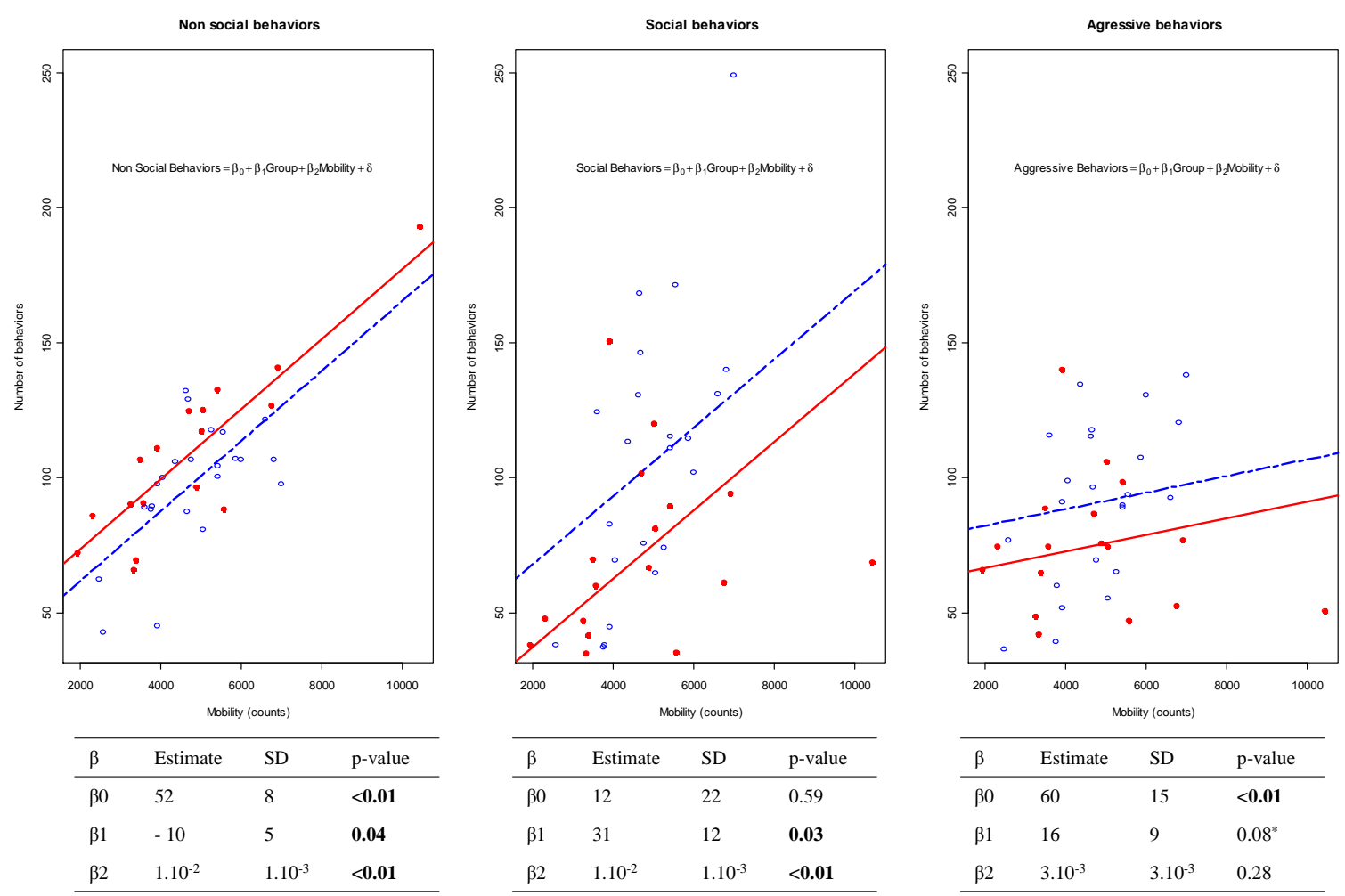

\begin{tabular}{llll}
\hline$\beta$ & Estimate & $\mathrm{SD}$ & $\mathrm{p}$-value \\
\hline$\beta 0$ & 60 & 15 & $<\mathbf{0 . 0 1}$ \\
$\beta 1$ & 16 & 9 & $0.08^{*}$ \\
$\beta 2$ & $3.10^{-3}$ & $3.10^{-3}$ & 0.28 \\
\hline
\end{tabular}

Figure 2: A general linear model was performed to adjust upon motility (spontaneous activity in lower position in the open field) the differences observed between lesioned and sham operated rats concerning social interaction tests.

Red/full circles: lesioned rats; Red lines: predicted values obtained from the general linear model in the lesioned rats;

Blue/empty circles: sham rats; Blue/dashed lines: predicted values obtained from the general linear model in the lesioned rats.

$\beta 0$ : intercept; $\beta 1$ : effect of the SHAM group (reference= lesioned); $\beta 2$ : effect of mobility.

* Trend to statistical significance 



Figure 3: Measures of elevated plus-maze conventional and ethological parameters. Data are expressed as boxplots for lesioned $(n=19)$ and sham lesioned $(n=26)$ rats. The lower and higher boundaries of the box indicate the 25th and 75th percentiles. The line within the box marks the median, and error bars below and above the box indicate the 10th and 90th percentiles.

$\star$ Statistically significant results

Trend toward statistical significance 